\title{
Study on blasting vibration response mechanism of talus slope based on particle discrete element model
}

\section{Dapeng Zhu}

Southwest Petroleum University

Changjian Xie ( $\nabla$ xcj_swpu@163.com )

Southwest Petroleum University https://orcid.org/0000-0003-0987-4137

\section{Latie Abu}

Southwest Petroleum University

\section{Research Article}

Keywords: particle discrete element model, talus slope, blasting vibration, attenuation law, Sadovsky formula

Posted Date: September 28th, 2021

DOI: https://doi.org/10.21203/rs.3.rs-934445/v1

License: (c) (i) This work is licensed under a Creative Commons Attribution 4.0 International License. Read Full License 


\title{
discrete element model
}

\section{Dapeng $\mathrm{Zhu}^{1,2} \cdot$ ChangjianXie $^{1} \cdot$ Latie $\mathrm{Abu}^{1}$}

\begin{abstract}
Talus slope are widely distributed all over the world, which is a special natural landscape and widely distributed in southwest and Northeast China particularly. It's formed by the collapse and transportation of parent rock, freeze-thaw cracking and other reasons, so it's often loosely accumulated at the foot of the hillside or at the gentle hillside. They are usually composed of collapsed rockfalls and weathered soil mixtures with large particle sizes. Therefore, Tunnel excavation construction through blasting will directly threaten the construction and operation safety of Railway, Highway and other projects along the way, which is very easy to cause heavy economic and personnel losses. In order to explore the dynamic response mechanism of overlying loose talus slope affected by blasting construction, taking the blasting excavation of Daqianshiling talus slope as an example, we measured the size of rocks on the slope on site, carried out the on-site and indoor geotechnical tests, and monitored the velocity and acceleration in real time. Then, we constructed the two dimensional particle discrete element model of irregular rock under blasting, which calibrated based on macro mechanical parameters obtained from field measurements. On this basis, we studied the process of rock breaking by blasting, the dynamic response law of slope, the propagation law of stress wave reflected and an interesting phenomena, eddy current phenomenon, by the the numerical simulation. Finally, we verified the applicability and limitation of Sadovsky family formulas for talus slope under blasting. This research is conducive to controlling blasting parameters and safe construction.
\end{abstract}

Key words: particle discrete element model; talus slope; blasting vibration; attenuation law; Sadovsky formula

Changjian Xie

Dapeng Zhu

xcj_swpu@163.com

1 School of Geoscience and Technology, South west Petroleum University, Chengdu 610500, China

2 Key Laboratory of Geological Hazards on Three Gorges Reservoir Area (China Three Gorges University), Ministry of Education, Yichang, Hubei 443002, China 


\section{Introduction}

As a special geomorphic landscape, talus slopes (Wang et al. 2014) are widely distributed in southwest and Northeast China. They're formed by collapse and transportation of parent rock, freeze-thaw cracking and other reasons. They're often loosely stacked at the foot of hillside or gentle hillside, which directly threatens the construction and operation safety of railway, highway or other projects along the way. They're a special adverse geological disaster. Major geological disasters are easy to cause heavy economic and personnel losses (Shugar et al. 2021). When the tunnel crosses the talus slope to the underlying hard bedrock, it is difficult for large equipment to enter the site due to the geographical location of the mountain area. Therefore, it's often used for construction that the method of grouting consolidation in the tunnel entrance and a certain range above it, and then blasting excavation (Zhu and Xie 2020), so as to ensure the safety of the slope and the construction period at the same time.

Excessive explosive mass used in blasting will cause violent vibration, tumbling and jumping of rock, and even block in the tunnel entrance, affecting tunnel construction and operation. The vibration response mechanism of talus slope subjected to blasting vibration is unclear, the propagation law of stress wave is unknown, and there is no mature evaluation method for reference. Therefore, it is necessary to study the vibration response mechanism of talus slope under blasting.

The existing monitoring equipment (such as blasting vibrometer) can't be placed into the slope, and must be fixed on the rock block to maintain synchronous vibration with it. It's difficult to monitor the inside. Because the talus slope has no constraints to restrict the movement of rock blocks, it is easier to move downward after vibration, therefore the harm is greater. Consequently, the monitoring and evaluation of vibration intensity is mainly based on the vibration velocity of rock blocks on slope, and further research is carried out with the help of numerical simulation. If the vibration of loose geotechnical medium such as talus slope is regarded as the collision between particles, Hertz's Law (Hertz 1881) is quite different because the friction between particles is not considered. The collision process between particles is very complex, and there are some shortcomings in field and laboratory tests, such as high cost and long time-consuming. The relevant theories are not widely used. For instance, the Sadovsky formula commonly used in China (Sadovsky 1945) is relatively less used in talus slope. On the basis of meeting the actual physical conditions, numerical simulation can accurately represent the static and dynamic process of geotechnical media.

The existing numerical simulation methods related to the study of dynamic response mechanism of rock masses are mainly based on the continuous and discontinuous medium theory. The former includes the finite element method $(\mathrm{Xu}$ 2017) and the finite difference method (He 2017). The latter includes particle discrete element model (DEM) and discontinuous deformation analysis (DDA), which is rarely applied to the study. Due to the discontinuity and local or global anisotropy of the talus slope, the numerical model based on the continuum theory has a large deviation in the mechanical behavior, such as the velocity change of a particle and the phase change characteristics of the overall flow (Zheng et al. 2021). The DEM based on Newton ' s second law (Cundall and Strack, 1979; Scaringi et al. 2018) has better applicability (Xu et al. 2019), which enable qualitatively or semi-quantitatively to analyze the propagation law of waves in granular materials such as talus slope (Wang 2020). At the same time, Nabipour (2013) and Hazzard (2004) verified the rationality of stress wave propagation by using 2D and 3D DEM respectively, and confirmed the applicability of DEM to simulate dynamic processes such as blasting. However, there are also shortcomings such as large amount of calculation, especially engineering level, and difficulty in modeling (Shi et al. 2021), so it is necessary to carry out targeted research.

At present, there are few studies on the vibration response mechanism and attenuation law of talus slope blasting based on DEM in China and abroad. Moreover, the existing numerical simulation is mostly based on the theory of continuous medium, without considering the characteristics of discontinuous dynamic response of rock blocks, especially the irregular shape and the stress wave response law at the interface of different geotechnical media. Therefore, on the basis of field measurement, geotechnical test and vibration monitoring, we constructed the 2D DEM of the Daqianhsiling talus slope blasting vibration, and calibrated the microscopic parameters to study the vibration response mechanism, attenuation law of velocity, and so on. Deep understanding of the vibration response mechanism of rock under blasting is helpful to maintain slope stability and economic and safe construction. 


\section{Overview of the study area}

\subsection{Engineering overview}

Daqianshiling is located in the eastern section of Tianshifu-Huanren Railway with a slope of $20 \sim 40^{\circ}$. The tunnel passes through the slope about $126 \mathrm{~m}$, and the maximum buried depth is about $50 \mathrm{~m}$. The main component of the slope is Quaternary collapse, Quartz sandstone of the Sinian Diaoyutai Group with grey-shallow grey, block, gravel and silty clay, which can be divided into the talus, accumulation horizon, diluvium and underlying bedrock. Each layer is loosely stacked above the next. The tunnel passes through the slope from the elevation of $435 \mathrm{~m}$, and the engineering geological profile of the slope at the entrance is shown in Fig. 1. Considering the loose structure, high porosity and poor stability of the talus slope, the sulphoaluminate cement mortar grouting is adopted to form the cemented body with high strength. The grade of the surrounding rock is improved from VI to V. In order to ensure safe operation and reduce the disturbance of blasting on rock, the excavator and crushing hammer are used as much as possible in the import section to cooperate with tunneling. The microseismic controlled smooth blasting technology is adopted when blasting operation is required.

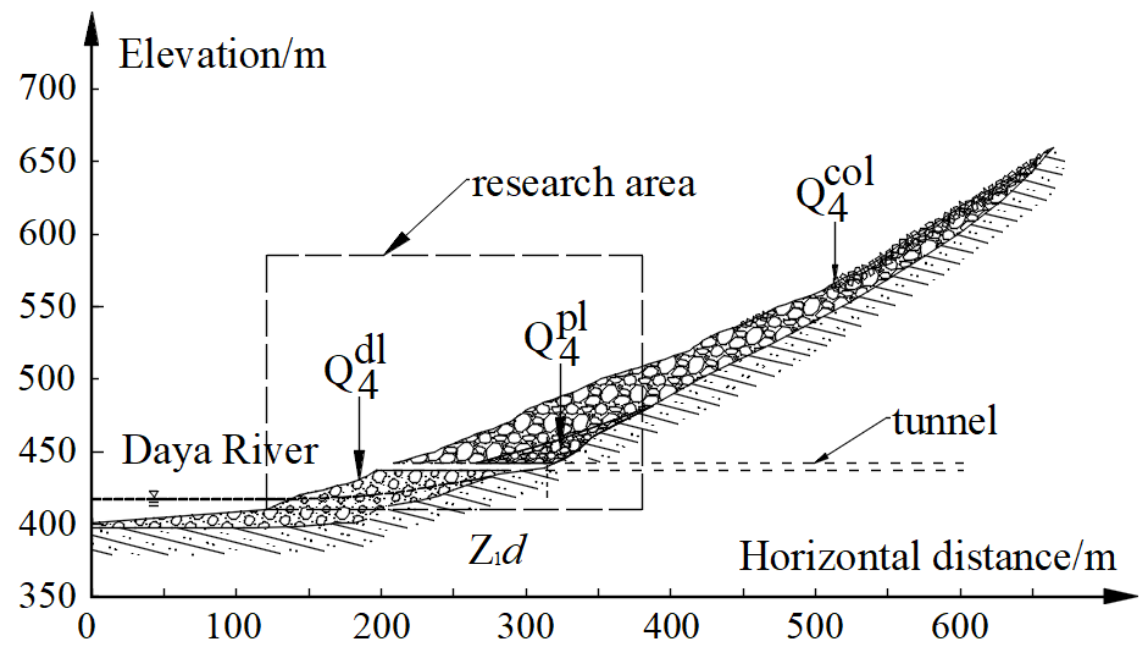

Fig. 1 Engineering geological profile of talus slope

\subsection{Characteristics of particle size distribution of rock and soil}

(1) Talus

The large area of the slope at the entrance of Daqianshiling is covered by talus, and bare bedrock only appears at the top of the trailing edge of the slope. The surface of the talus at the entrance of the tunnel is covered with moss, no vegetation and humus, and most of them are long strip, conical and saddle-shaped. Rock size is mainly $0.3 \sim 1.2 \mathrm{~m}, 2.1$ $\mathrm{m}$ individually. Overall overhead structure, no broken stone and fine soil filling and the talus is overall overhead structure.

In order to obtain the size distribution of rock blocks, taking the tunnel entrance as the starting point, the size of them in the range of $480 \mathrm{~m}$ slope length is measured upward.

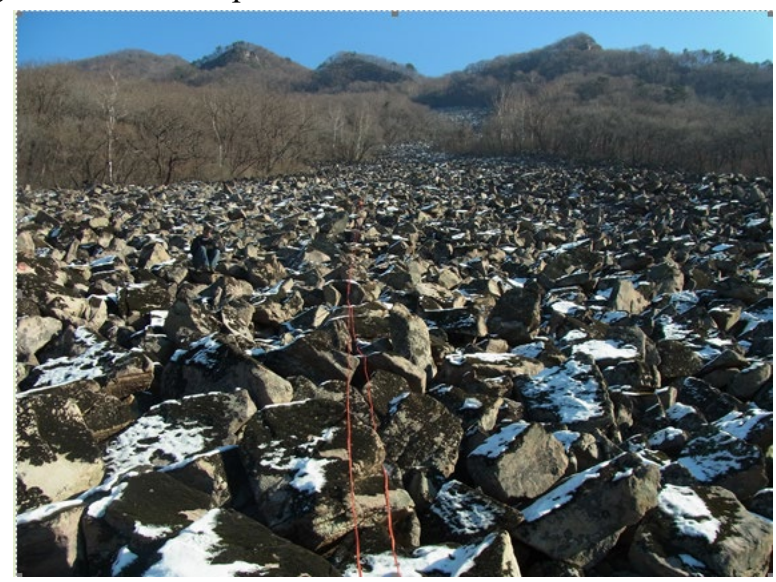

Fig. 2 Field measurement of rock size 
After smoothing by Savitzky-Golay method, the distribution characteristics were observed. The characteristic of this method is that it can ensure the distribution shape and width of the value are unchanged. From Fig. 3, it can be seen that the overall size of rock blocks does not change significantly with slope height. The size of rock blocks at toe and top of slope is relatively small.

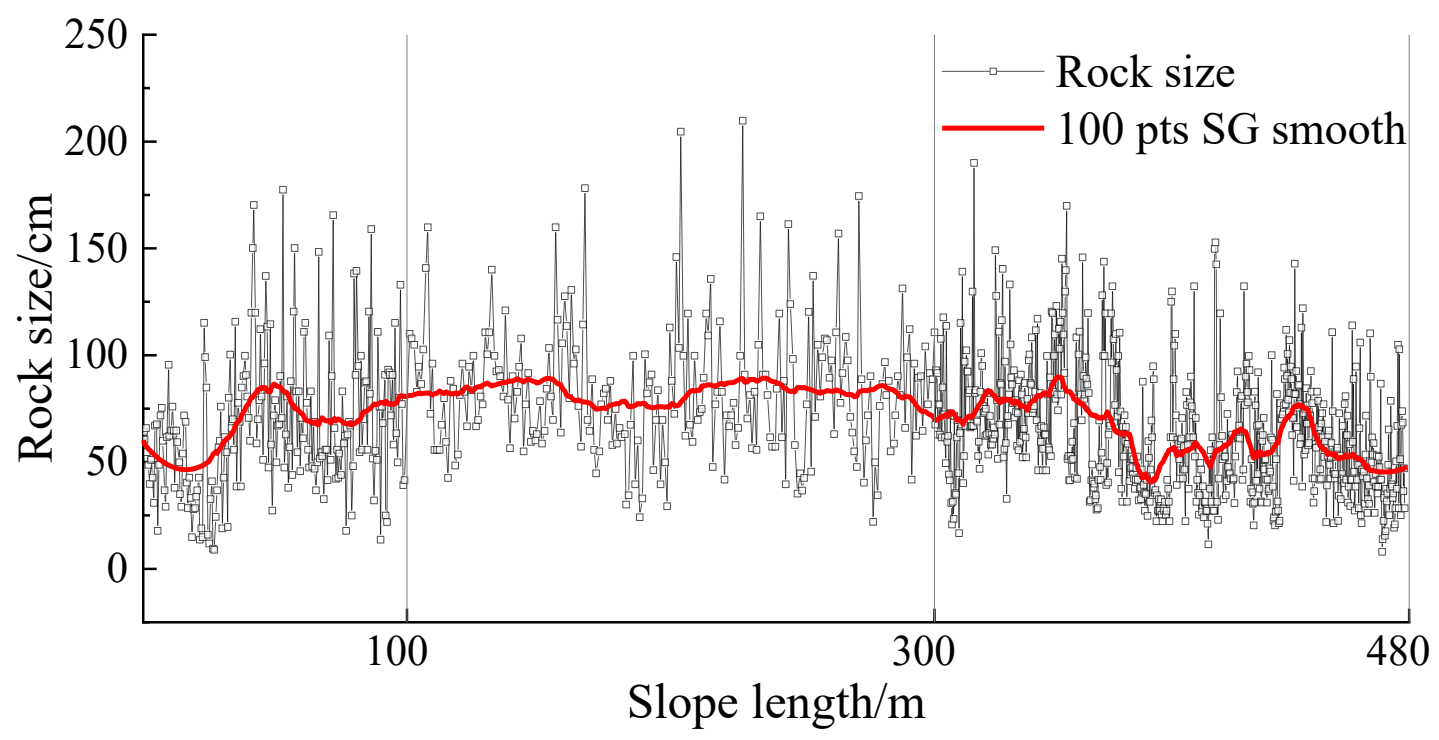

Fig. 3 Size distribution of rock

We convert the rock size by JOHNSON conversion, and the conversion formula is as follows:

$$
d_{\text {con }}=2.49+2.03 \times \ln \frac{d_{\text {orig }}+15.46}{336.84-d_{\text {orig }}}
$$

where, $d_{\text {orig }}$ and $d_{\text {con }}$ are the original size of the rock and the transformed one, $\mathrm{cm}$, respectively. After conversion, the average size is 0.002 and the standard deviation is 1.004 .

The Kolmogorov-Smirnov test of single sample shows that when the confidence level is $95 \%$, the significance level is 0.056 , greater than 0.05 . That is, the converted rock size is significantly in line with the Gaussian distribution. The macroscopic confusion is only external performance.

(2) accumulation horizon and diluvium

The accumulation horizon and diluvium are mainly composed of yellow and gray-white, loose sub-angular, stonegravel soil, which are moderately weathered. The composition is shown in Table 1. The block stone supports the whole skeleton structure in the form of natural overhead accumulation. The gravel and sand are tightly filled with silty clay without weak interlayer. The diluvium is dominated by gravel soil, and rare large granular stones. Uneven coefficient of gravel soil, $C_{u}$ is 29.6, curvature coefficient, $C_{c}$ is 1.3. It's graded continuous heterogeneous soil.

Table 1 shape and size of geotechnical materials in accumulation horizon

\begin{tabular}{cccc}
\hline Type & shape & Size $/ \mathrm{m}$ & $\begin{array}{c}\text { volume } \\
\text { ratio }\end{array}$ \\
\hline Block & blocky & $0.5 \sim 1.2$ & $35 \%$ \\
Gravel & suborbicular & $0.2 \sim 0.5$ & $30 \%$ \\
Sand & grainy & $<0.02 \sim 0.2$ & $25 \%$ \\
\hline
\end{tabular}

\subsection{Blasting layout}

The blasting adopts non-electric millisecond detonator and smooth blasting technology, and the charge decoupling coefficient is $1.4 \sim 2.0$. 2\# rock ammonium nitrate explosives were used for the blasting. All blastholes were detonated almost at the same time, therefore the influence of single charge and blasthole diameter of each blast can be ignored. Sum up the charge of each hole as the charge of each blasting, and 180 blastholes were arranged. In addition to the change of 
dosage, the rest such as type, depth of charge, charge mode and hole arrangement are basically the same (Qi 2009), which can be regarded as only the change of total dosage $Q$.

\section{Response law of talus slope under field blasting}

The acceleration and velocity of rock slope are affected by the spatial orientation, propagation medium and explosive factors, such as distance between blasting center and monitoring point, $R$, elevation difference, $H$, and dosage, $Q$. The field measured acceleration is shown in Fig. 4.

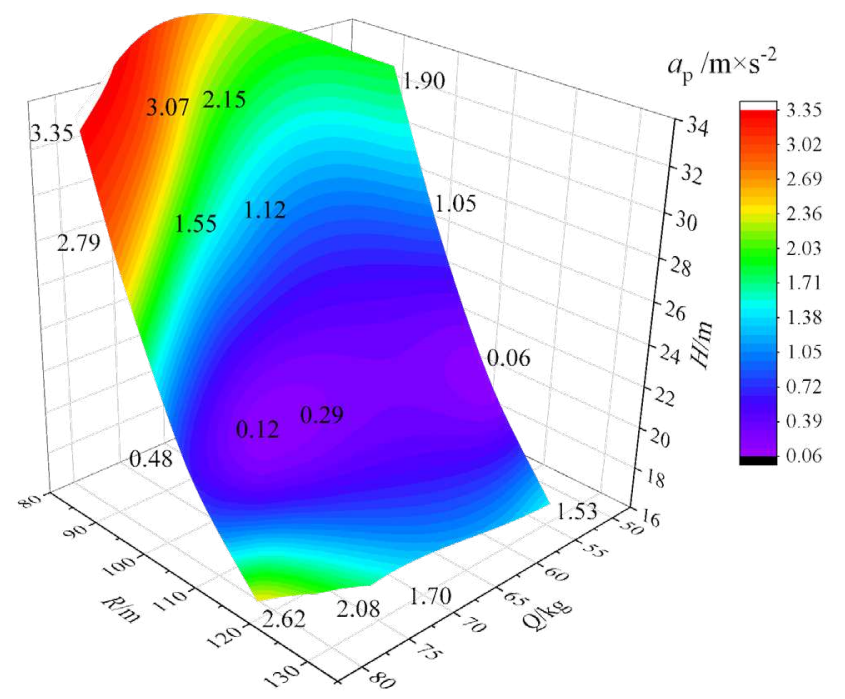

Fig. 4 Diagram of peak acceleration, $a_{\mathrm{p}}$, under different $R, H$ and $Q$

From Fig. 4, it is found that there is not a simple linear relationship between the peak acceleration, $a_{\mathrm{p}}$, peak velocity, $v_{\mathrm{p}}$, and $R, H$, and $Q$. The peak acceleration and peak velocity are inversely correlated with $R$, and positively correlated with the $Q$, and there is amplification effect at tunnel entrance. Due to the limited monitoring number, the higher and the interior of the slope, whether it satisfies is unknown.

\section{Collision and fragmentation of rock}

In the process of rock collision, the contact point produces large local high strain rate and stress concentration. At the same time, the stress wave oscillates and propagates along the surface and interior of the rock, and it is enclosed in the rock in the form of vibration wave (Zener 1941) and causes energy loss. In the propagation process of blasting stress wave, it mainly experiences four consumption processes: local strain, internal elastic wave, particle kinetic energy and friction heat generation, which runs through the whole process of rock collision and vibration, and gradually dissipates the stress wave. In addition to blasting stress wave, there are kinetic energy of gravitational potential energy transformation of rock as energy input to participate in the energy transfer process.

In order to illustrate the principle of collision and fragmentation, two-dimensional elastic forward collision is taken as an example. The rock particles are equivalent to a circle. The mass of the two blocks in the plane is $m_{l}$ and $m_{2}$, the initial velocities are $v_{10}$ and $v_{20}$, and the velocities after collision are $v_{1 l}$ and $v_{2 l}$, respectively. Since the collision process is very short, the system is not affected by any external force (such as gravity), so it satisfies the law of conservation of momentum. Because the collision between rock blocks is between complete elasticity and complete inelasticity, the recovery coefficient $e$ is introduced, which between 0 and 1. It can be determined according to the uniaxial compressive strength, elastic modulus and equivalent radius (Hu and Liu 2003).

Let $m_{l}=k m_{2}$, where $k$ is the mass ratio, then:

$$
v_{11}=v_{10}-\frac{k(1+e)}{1+k}\left(v_{10}-v_{20}\right) \quad \text { (2) }
$$




$$
v_{21}=v_{20}+\frac{1+e}{1+k}\left(v_{10}-v_{20}\right)
$$

According to the conservation law of kinetic energy, the kinetic energy loss, $\Delta T$, is obtained as follows :

$$
\Delta T=\frac{k\left(1-e^{2}\right)}{2(1+k)}\left(v_{10}-v_{20}\right)^{2}
$$

The material of rock mass is the same, so the volumetric strain energy of the two rock masses is equal. According to the theory of volumetric strain energy intensity, the strain energy of rock mass broken by collision should satisfy :

$$
U_{1}=U_{2}=\frac{1}{2} \Delta T=\frac{k\left(1-e^{2}\right)}{4(1+k)}\left(v_{10}-v_{20}\right)^{2} \geq \frac{\sigma m}{2 E \rho}
$$

where $U_{1}$ and $U_{2}$ are elastic strain energy of two rock blocks respectively. $E$ is elastic modulus, $\rho$ is density, $m$ is mass, and $\sigma$ is uniaxial compressive strength. When the strain energy satisfies formula (5), the rock will fragment. If not satisfied, it mainly causes the internal crack propagation.

Due to the loose accumulation of rock blocks, the more general case should be oblique collision, and the problem of collision between rock particle chains should also be considered. If the tangential friction between rock masses is not considered, the tangential component of velocity should also meet the law of conservation of momentum. So oblique collision can be transformed into forward collision. The tangential friction mainly generates friction heat and causes rigid body rotation, and the friction coefficient and rock shape are related to the energy conversion process ( $\mathrm{Hu}$ and Liu 2003). When the rock mass collides with the bedrock, the contacts with the bedrock surface is disordered, and the blocks are filled with gravel and fine-grained sand. The dynamic collision and energy conversion process are extremely complex, which need to be further studied by numerical simulation.

\section{2D particle discrete element model construction}

The talus slope is obviously discontinuous, and the shape, size and distribution characteristics of blocks are quite different, so it is impossible to determine the macroscopic mechanical characteristics directly by in-situ test or direct shear test. The FEM and FLAC which are suitable for simulating continuous medium can't accurately simulate the unique constitutive relationship of loose blocks, and can't show the dynamic response process of discontinuous large deformation of talus slopes. The DEM uses a sphere to simulate the contact relationship, and simulates the mechanical properties of geotechnical media through the combination of particles. Based on the measured results of field blasting, the DEM of talus slope at the entrance of Daqianshiling is constructed to analyze the dynamic response process.

\subsection{Particle construction of irregular rock}

The stability of rock mass depends on shear strength, which is mainly affected by block shape, surface friction coefficient and other factors (Edmans and Sinka 2020). The wave velocity of soil-rock mixture is mainly affected by void ratio, elastic modulus and particle shape (Zhang Fei et al., 2020). Therefore, the change of rock block shape can't be ignored in the study of dynamic response process. Although the shape of rock mass is diverse and the spatial position is uncertain, the size diversity and complexity similar to that of nature can still be obtained by randomly simulating its surface characteristics on the basis of simplifying the surface size. Spherical particles cannot consider strong bite force (Cho et al. 2010), but irregular particles can reflect the influence of irregular shape of rock blocks on bite force (Bahrani et al. 2011).

The randomness of rock shape is mainly affected by the randomness of the number, radius and angle of surface control points for a single rock. On the whole, the randomness of the overall rotation should also be included. Therefore, according to the above investigation of rock mass size, the rock mass with Gaussian distribution after rock mass size correction is constructed. Assuming that the randomness of rock control points conforms to Gaussian distribution too ( $\mathrm{Su}$ and Wang 2021), 64 templates are constructed as shown in Fig. 5. When the mean value of control points is 7 and the variance is 2.0 , the rock block shape obtained is basically consistent with the rock block shape in the field.

In addition, the spatial distribution characteristics of the talus should be considered in the field simulation. The 
correlation between rock block size and elevation is weak. It is assumed that the spatial position and rotation angle of rock blocks with different sizes conform to the uniform distribution. Firstly, the volume of rock mass generates regular sphere (volume $V_{2}$ ) according to the same gradation curve as the field rock mass (volume $V_{l}$ ), and then the equal volume replacement method is used to replace the irregular sphere (volume $V_{3}$ ) to obtain the distribution characteristics of rock mass that have been in the field. Because it's irregular, the sphere volume obtained is larger than the actual rock block, namely $V_{1}<V_{2}$. Under equal volume replacement, $V_{2}=V_{3} . V_{l}=\eta^{2} V_{2}=\pi \eta^{2} r^{2} . \eta$ is the volume equivalent coefficient. when $\eta$ is 0.85 , it has a good fitting effect that the test shows.

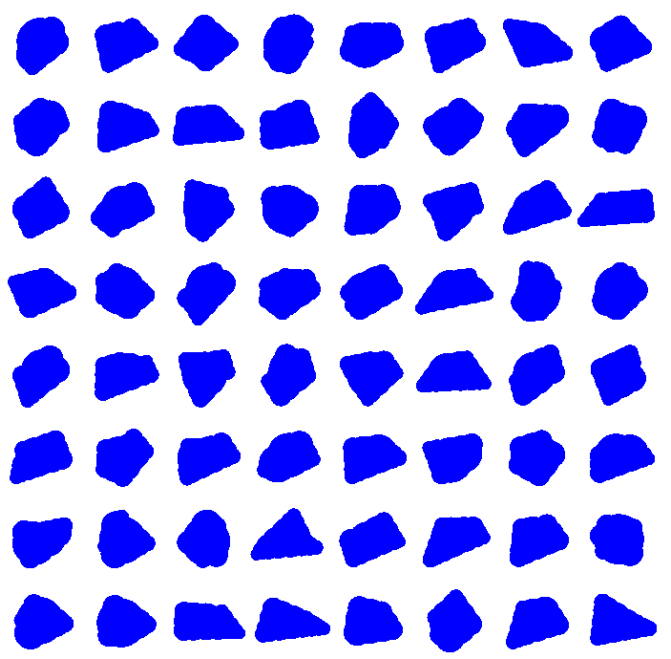

Fig. 5 Irregular template of rock in talus

In addition to the rock in talus, the rock in the accumulation horizon, diluvium and underlying bedrock is regarded as regular circular due to high roundness.

\subsection{Calibration of microscopic parameters}

The rock-soil-gravel soil in the accumulation horizon is mixed with large stones. Relying on the indoor large-scale direct shear instrument to carry out the direct shear test will obtain the results that can reflect the actual situation better than the conventional small-scale direct shear test, which eliminate the coarse particles and affect the test results. We test the macro parameters of diluvium through indoor small-scale direct shear test and the one of bedrock and talus through uniaxial compression test.

There are three main problems in the construction of 2D slope, the selection of particle and model size, the porosity conversion of $3 \mathrm{D}$ to $2 \mathrm{D}$ and the calibration of microscopic parameters. The quartz sandstone particles are in the millimeter level. If the small particles are used to build the engineering level model, the number of particles will inevitably increase. Therefore, the step-by-step verification method (Peng et al. 2020) is used to gradually adjust the parameters, so that under the same mechanical conditions, the macroscopic parameters of the numerical model are basically consistent with the macroscopic parameters of the laboratory test. It can be considered that the numerical model meets the requirements of size and strength. The specific steps are as follows:

(1) Firstly, we established a numerical model (Model 1) with the same size and particle radius as the rock sample in the laboratory test. Through the numerical test and the trial-and-error method, the microscopic parameters that were basically consistent with the mechanical behavior of the laboratory test were gradually obtained. (2) Then, Keeping the parameters and particle radius unchanged, we expanded the size of numerical model (Model 2), and obtained the mechanical parameters through numerical experiments. (3) Finally, Keeping the numerical model size consistent with the model two, we expanded the particle radius (model 3), and adjusted the microscopic parameters to keep consistent with the mechanical behavior of model 2. Repeat step (2) and step (3) to obtain the appropriate particle size, the microscopic parameters can be used to establish the engineering level model.

The measured porosity is three-dimensional, and the corresponding two-dimensional porosity needs to be converted. Particle structure is not regular structure such as hexahedron and hexagonal lattice structure (corresponding to the loose 
and dense state of particles with equal particle size, respectively) (Wang et al. 2014). The relative density factor, $D_{r}$, is introduced to obtain two-dimensional porosity:

$$
n_{3 D}=1-(0.25 D r+0.61)\left(1-n_{2 D}\right)^{1.5}
$$

where $n_{3 D}$ and $n_{2 D}$ are the three-dimensional and two-dimensional porosity of the sample, respectively. The approximate two-dimensional porosity can be obtained by this formula. Since the size of the talus and accumulation horizon is known, it can be generated directly. The bedrock and diluvium are not the focus, only to meet the strength requirements. The microscopic parameters of different rock and soil are calibrated separately according to numerical tests.

(1) Bedrock

In order to simulate the failure characteristics of rock mass at high strain rate, the strength and stiffness parameters of rock were obtained by the conventional uniaxial compression test under different strain rates for the rock samples taken in the field, and then calibrated according to the numerical test.

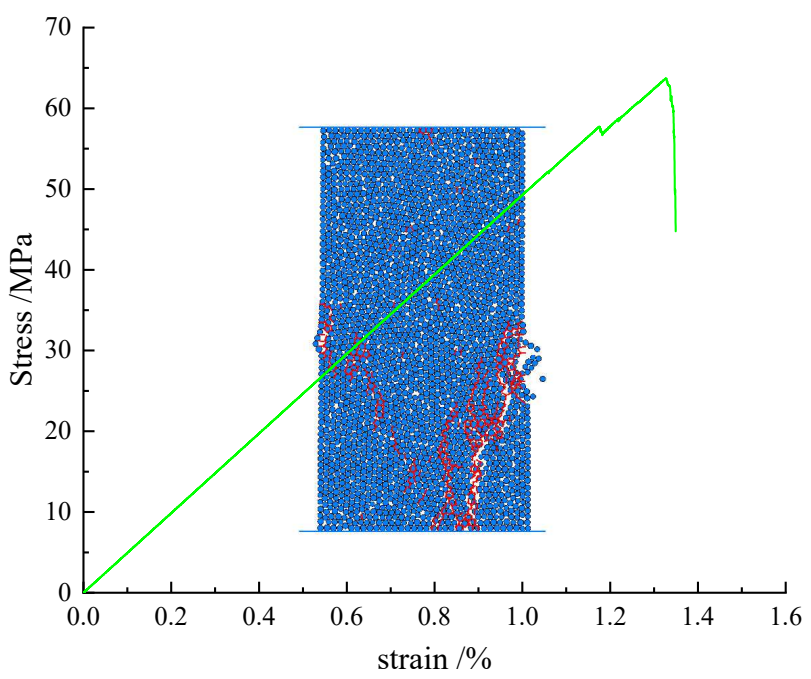

Fig. 6 Numerical uniaxial test

The simulated rock sample size is $15 \mathrm{~m} \times 30 \mathrm{~m}$, and the particles are uniformly distributed in the range of $0.18 \sim$ $0.25 \mathrm{~m}$. The minimum size ratio of the model to the minimum size of the particles is 41 . The porosity of the model is 0.10 and 2758 particles are generated. The contact is modeled by linear parallel bond model. Under static load, the macroscopic elastic modulus $E$ is $5.03 \mathrm{GPa}$, the compressive strength is $75.33 \mathrm{MPa}$, and the Poisson ' s ratio is 0.24 , which is basically consistent with the actual parameters of samples.

The dynamic stiffness and dynamic strength of rock mass should also be considered in the process of blasting dynamic action. The ratio of them to strain rate $\varepsilon_{k}$ under dynamic and static load satisfies (7) and (8).

$$
\begin{aligned}
K_{d} & =\left[a \lg \left(\varepsilon_{k}\right)+b\right] K_{s} \\
\sigma_{d} & =\left[c \lg \left(\varepsilon_{k}\right)+d\right] \sigma_{s}
\end{aligned}
$$

Where a $\sim \mathrm{d}$ is coefficient. $K_{d}, K_{s}, \sigma_{d}$, and $\sigma_{s}$ is dynamic stiffness, static stiffness, dynamic strength and static strength respectively. Through the conventional uniaxial tests with different strain rates $\left(1 \times 10^{-2}, 1 \times 10^{-3}, 1 \times 10^{-4}, 1 \times 10^{-5}\right.$ and $1 \times 10^{-}$ ${ }^{6}$ ), $a \sim d$ is $0.1434,1.0953,0.1206$ and 1.024 , respectively.

(2) Diluvium

The diluvium contains clay particle, therefore it's calibrated by numerical direct shear test. The size of shear box is $15 \mathrm{~m} \times 15 \mathrm{~m}$, and the contact model is linear parallel bond model. From fig. 7, it can be seen that the shear stress and shear displacement curves simulated under different normal stresses are well fitted. Since the shear stress has no peak value, it is considered that the disturbed soil sample has been sheared when the reading slowly increases to the level and the shear deformation increases sharply, and the stable value is taken as its shear strength. The values of $c$ and $\varphi$ obtained by linear fitting are $10 \mathrm{kPa}$ and $29^{\circ}$, respectively. 


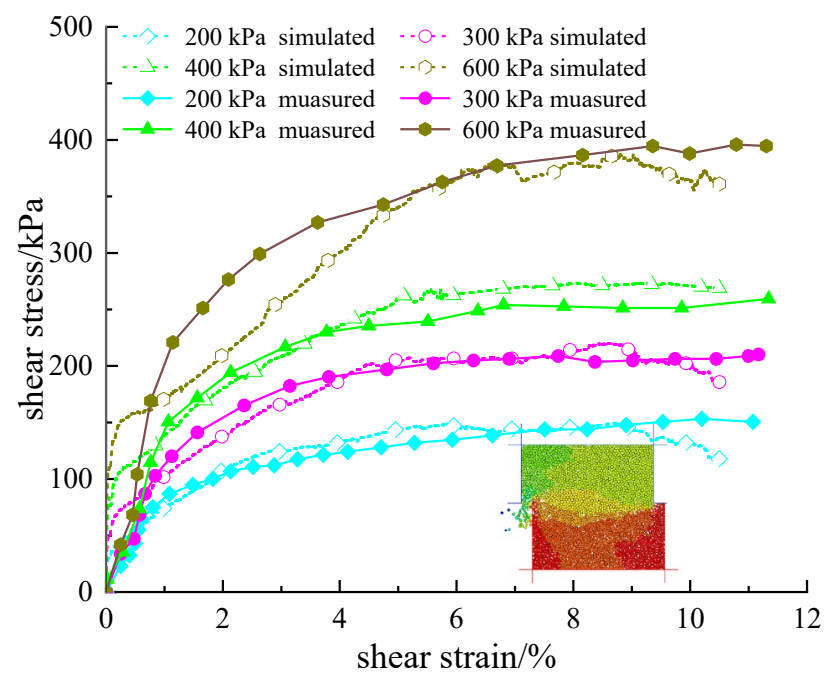

Fig. 7 Numerical direct shear test

(3) Talus and accumulation horizon

The internal frictional angle is obtained by the natural angle of repose. Using the test method of simulating natural accumulation, the rock blocks are naturally accumulated directly in the container then slowly lifting the container.

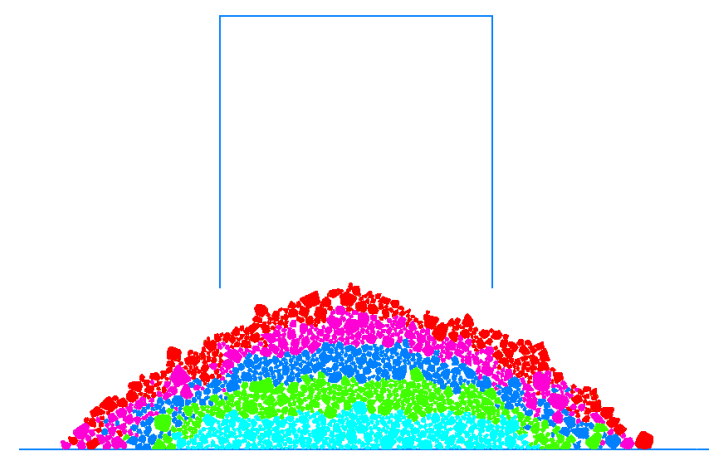

Fig. 8 Numerical accumulation test

Using the above irregular particle generation method, there are 830 symbiotic diagenetic blocks with a size range of $0.3 \sim 2.1 \mathrm{~m}$. Due to the characteristics of loose accumulation and large porosity, the bond strength and cohesion between particles can be ignored. The main physical parameters are the weight density, stiffness and internal frictional angle. The final accumulation results are shown in Fig. 8. The same microscopic parameters are used in the accumulation horizon.

In summary, the micro-mechanical parameters of each layer are shown in Table 2. Linear contact is adopted at the interface of each layer, and the contact stiffness and strength are half of the medium on both sides. In order to prevent tunnel collapse, the lining is equivalent to a multi-section wall, and the wall is added and balanced after tunnel excavation. The vertical increment $\Delta F_{y}$ of the force exerted by the rock mass on the lining after blasting will cause the lining to move in the same direction. If lining is regarded as linear elastic material, there are:

$$
\Delta F_{y}=K_{e} \Delta S=E L \times \Delta v \times \Delta t
$$

Where $K_{e}$ is the normal stiffness of the lining, GPa. $\Delta S$ is the numerical displacement increment of lining, $L$ is the length of lining, $\Delta v$ is the vertical increment of velocity obtained by lining. If $\Delta t$ is updated once in each time step, it's a constant. The equation (9) indicates that $\Delta v$ obtained by lining is proportional to the vertical increment of contact force. It should be noted that the linear parallel bond model is adopted between rock and lining to make them not separate.

Table 2 Macroscopic parameters of rock-soil mass

\begin{tabular}{|c|c|c|c|c|c|c|}
\hline \multirow[t]{2}{*}{ name } & Bulk density & shear modulus & poisson's & ratio cohesion & $\begin{array}{c}\text { frictional } \\
\text { angle }\end{array}$ & porosity \\
\hline & $\mathrm{kN} \times \mathrm{m}^{-3}$ & $\mathrm{GPa}$ & / & $\mathrm{kPa}$ & $\circ$ & $\%$ \\
\hline talus & 21.0 & 0.019 & 0.30 & 20 & 35.0 & 20 \\
\hline
\end{tabular}




\begin{tabular}{ccccccc}
$\begin{array}{c}\text { accumulati } \\
\text { on horizon }\end{array}$ & 21.0 & 0.019 & 0.30 & 20 & 35.0 & 20 \\
diluvium & 20.5 & 0.074 & 0.25 & 10 & 29.0 & 15 \\
bedrock & 26.0 & 2.08 & 0.20 & 1500 & 50.0 & 5 \\
\hline
\end{tabular}

Table 3 Micro-parameters of rock-soil mass

\begin{tabular}{ccccc}
\hline Mesoscopic parameters & Bedrock & Diluvium & Accumulation horizon & Talus \\
\hline Radius $/ \mathrm{m}$ & $0.18 \sim 0.25$ & $0.18 \sim 0.60$ & $0.18 \sim 0.60$ & $0.10 \sim 1.05$ \\
Shape & circle & circle & circle & random \\
Porosity & 0.135 & 0.135 & 0.15 & 0.2 \\
Density $/\left(\mathrm{kg} \times \mathrm{m}^{-3}\right)$ & 2600 & 2050 & 2090 & 2143 \\
Contact type & linear parallel bond model & & linear \\
Friction coefficient & 0.5 & 0.2 & 0.5 & 0.5 \\
Normal stiffness $/ \mathrm{GPa}$ & 2.3 & 0.011 & 2.3 & 2.3 \\
Linear stiffness ratio & 2.0 & 1.5 & 2.0 & 2.0 \\
Normal bond stiffness /GPa & 2.3 & 0.011 & $/$ & $/$ \\
Bond stiffness ratio & 2.0 & 1.5 & $/$ & $/$ \\
Tensile strength $/ \mathrm{MPa}$ & 21.84 & 0.01 & $/$ & $/$ \\
Internal frictional angle $/ 0$ & 50 & 29 & $/$ & $/$ \\
Cohesion $/ \mathrm{kPa}$ & $3.0 \times 10^{4}$ & 50.0 & & \\
\hline
\end{tabular}

\subsection{Blasting and boundary conditions}

The blasting process is simulated by the method of expanding the radius of the package. The position of the package is fixed, the radius is expanded to overlap with the surrounding rock particles, and the larger overlap makes the rock particles obtain the blasting force. Blasting is equivalent to triangular load. Rise time, $t_{r}$, total time, $t_{s}$, are etermined by formula (10) and (11):

$$
\begin{aligned}
& t_{r}=\frac{1}{7} Q^{-0.15}\left(\frac{r}{r_{b}}\right)^{(2-\mu) / 6} t_{s} \\
& t_{s}=\frac{84}{K_{c}}\left(\frac{r}{r_{b}}\right)^{(2-\mu) / 3} Q^{0.2}
\end{aligned}
$$

Where $K_{c}$ is the volume compression modulus of rock mass, GPa. $\mu, Q$ same as above. $r$ is the distance from the center of the borehole, $\mathrm{m} . r_{b}$ is the hole radius, $\mathrm{m}$. The formula shows that when the detonation pressure and period are fixed, the pressure on the rock around the borehole is triangular with time. According to the linear contact principle of particles, the blasting pressure can be applied to the rock around the borehole by reasonably controlling the radius expansion relationship of the blasting package.

When the charge is not coupled, the maximum pressure $p_{\max }$ when the impact pressure reaches the hole wall satisfies (Yang et al. 2019):

$$
P_{\max }=2 n \rho_{0} Q_{v}\left(V_{c} / V_{b}\right)^{3}=2 n \rho_{0} Q_{v} k^{3} \eta^{6}
$$

Where $\rho$ is explosive density, $Q_{v}$ is the explosive heat. $V_{c}$ and $V_{b}$ are explosive volume and chamber volume. $n$ is the increase multiple, $8 \sim 11 . k$ is the charge coefficient, the ratio of charge length to blasthole length, 0.83 . $\eta$ is the charge uncoupling coefficient, the ratio of charge radius to blasthole radius, 0.66 .

Under the linear contact theory, the radius and contact stiffness between the circular borehole and the surrounding rock satisfy:

$$
r_{\max }=2 \pi r_{0} p_{\max } / K_{n}
$$

Where $r_{0}$ is the original radius, $\mathrm{m} . K_{n}$ is the combined contact stiffness between particles, GPa. $r_{\max }$ is the maximum expansion radius, $\mathrm{m}$. 
Therefore, by combining the above equations (10) (13), the relationship $r(t)$ between the radius of the package and the time can be obtained. $\rho_{0}$ is $1000 \mathrm{~kg} / \mathrm{m}^{3}, Q_{v}$ is $4000 \mathrm{~kJ} / \mathrm{kg}, n$ is $10, r_{0}$ is $0.15 \mathrm{~m}$. Finally, the rise time of explosive, total time and blasting load are shown in Fig. 9.

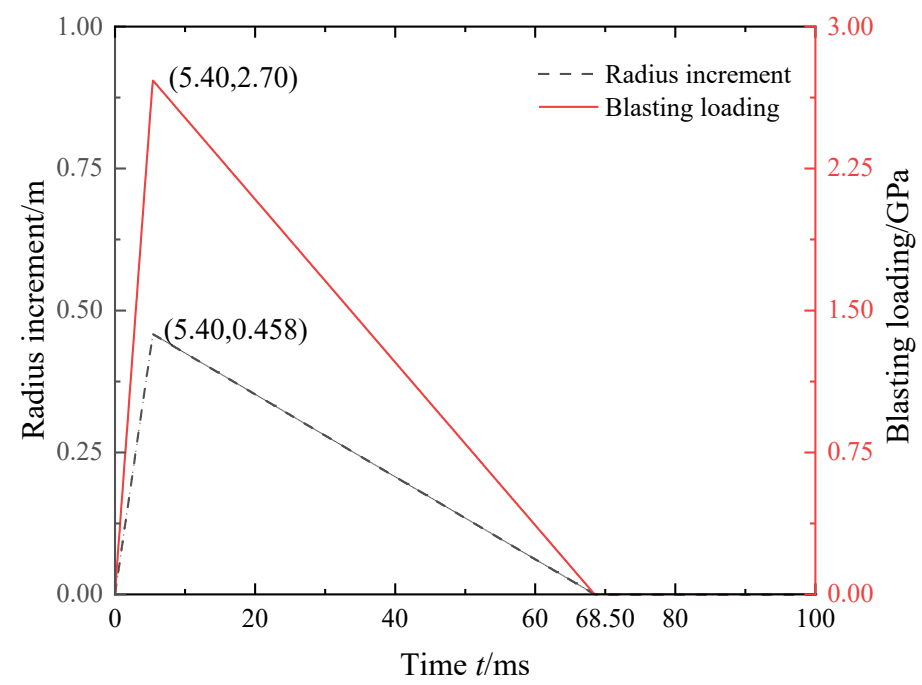

Fig. 9 Diagram of blasting triangular wave and radius increment

In order to simulate infinite medium, we need absorb the kinetic energy of incident wave or increase the transmission wave energy at the boundary. To improve the limitation of using high damping coefficient on the boundary, the boundary force is applied to the boundary particles and the boundary force is corrected by considering the dispersion effect of stress wave. The relationship between incident boundary force, transmission boundary force and particle velocity satisfies:

$$
F=\left\{\begin{array}{l}
-\xi_{n} \cdot 2 R_{p} \rho C_{P} v_{n} \\
-\xi_{s} \cdot 2 R_{p} \rho C_{S} v_{s}
\end{array}\right.
$$

Where $\xi_{n}$ and $\xi_{s}$ are correction coefficients of longitudinal wave and shear wave dispersion effect respectively. $R_{p}$ is the radius of particles, $\rho$ is the density of medium, $C_{p}$ and $C_{s}$ are $\mathrm{P}$ wave and $\mathrm{S}$ wave respectively. $v_{n}$ and $v_{s}$ are the normal and tangential velocities of particles, respectively. Both $\xi_{n}$ and $\xi_{s}$ are 0.35 , and the obtained stress wave effect is good.

If the stress wave propagation in non-boundary particles is realized by setting local damping, the velocity will be reduced and distorted at each calculation time step (Zhang et al. 2018). Therefore, the normal and tangential stiffness damping coefficients are set as 0.7 and 0.5 , respectively, to simulate the energy dissipation during particle collision.

\subsection{Establishment of DEM of the talus slope}

A total of 56336 particles and 18728 non-spherical particles were generated by using the shape, size, strength, stiffness and other parameters consistent with the calibration (Fig. 10).

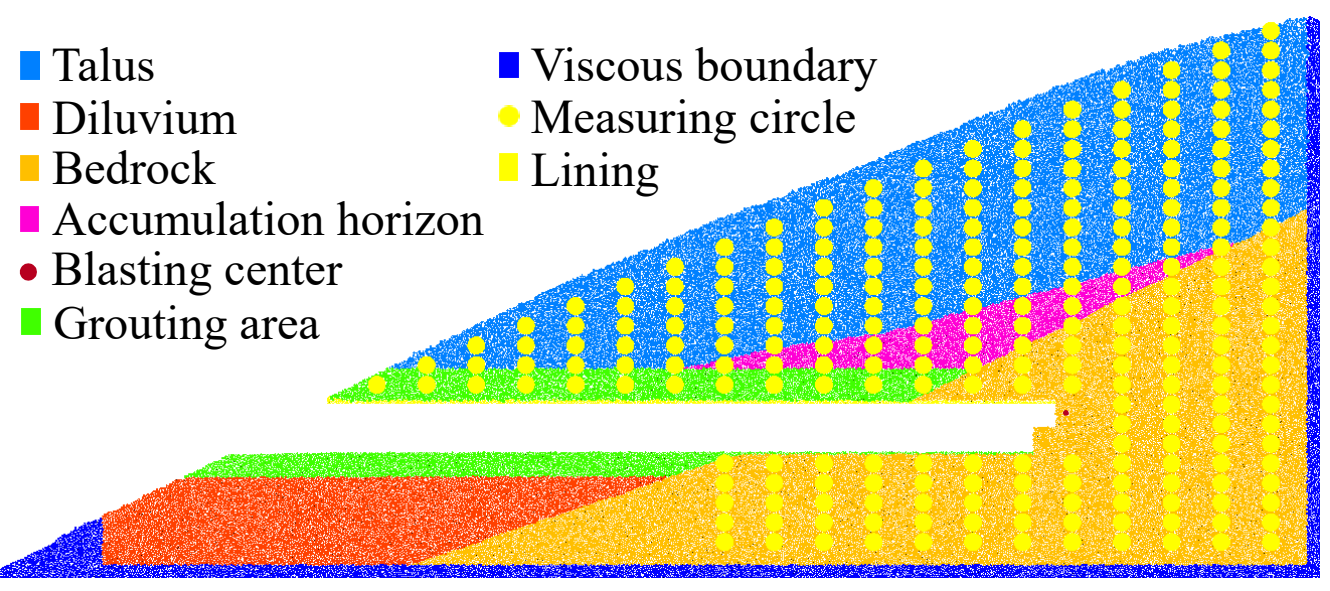

Fig. 10 DEM of Daqianshiling talus slope

The overall height of the model is about $130 \mathrm{~m}$ and the horizontal length is about $300 \mathrm{~m}$. The limit height of tunnel 
is $10.4 \mathrm{~m}$. The local coordinate system is established with the blasthole at the footage, $160 \mathrm{~m}$, as the origin. The direction to the tunnel entrance is the negative direction of $X$ axis, and the vertical direction is $Y$ axis. There are 262 monitoring points, with the horizontal interval $11.05 \mathrm{~m}$, the vertical interval $4.37 \mathrm{~m}$, and the monitoring radius $2.0 \mathrm{~m}$. The blasting center is set to the middle of the upper step face. When the maximum velocity is less than $1 \mathrm{~mm} / \mathrm{s}$, the model is considered to be balanced. The time step is $1.447 \times 10^{-5}$.

\section{Mechanism of slope vibration response under simulated blasting}

\subsection{Mechanism of Blasting Vibration and Stress Wave Propagation}

The high-temperature, high-pressure and high-speed gas generated by blasting rapidly acts on the rock of the blasthole and rapidly diffuses and attenuates outward, causing the vibration of the overlying slope. The whole process is mainly divided into three stages: shock wave, stress wave and seismic wave, which cause rock crushing, fracture and elastic vibration respectively (Fig. 11).

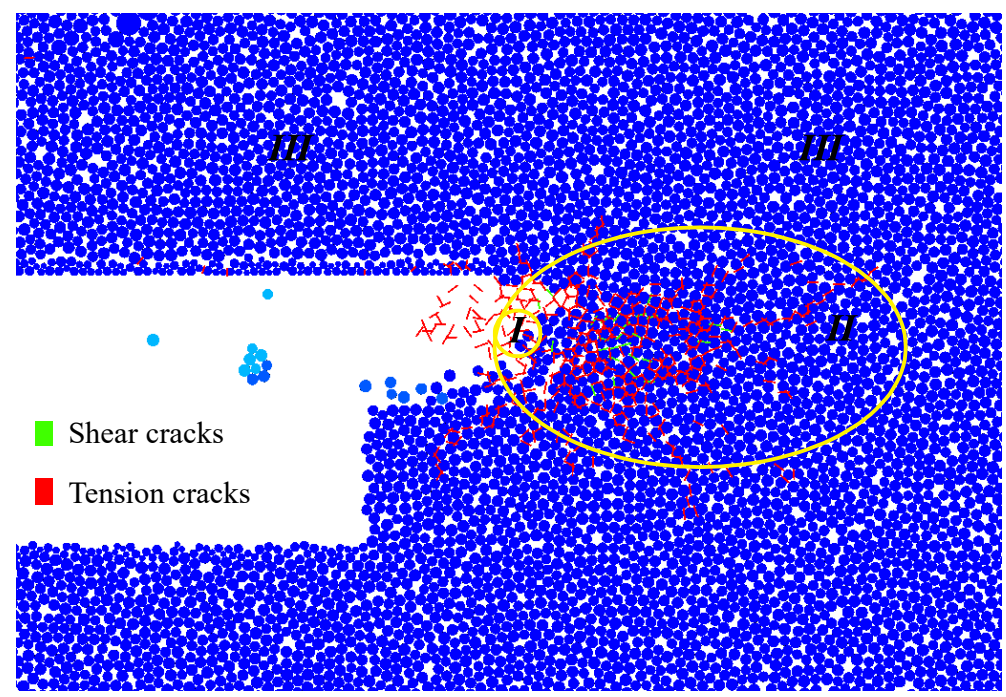

Fig. 11 Crack diagram near blast hole $\left(t=5.8 \times 10^{4}\right.$ steps; I, II and III represent crushing zone, fracture zone and elastic vibration zone respectively)

Stage 1, rock crushes around borehole (region I). At the initial stage of explosive detonation, the shock wave formed by detonation wave and detonation gas acts on the borehole sharply, and the peak pressure is much larger than the compressive strength of bedrock, which causes the bedrock to crush and form a crushing zone. Stage 2, rock mass rupture around borehole (region II). After the blasting shock wave is consumed through the crushing zone, it is rapidly attenuated to compressive stress wave, and the velocity of wave front is close to the sound velocity of rock mass. The rock mass produces radial compression deformation under radial compressive stress and radial cracks under tangential tensile stress. The radial initial fracture expands rapidly under the dual action of tangential tensile stress and air wedge. Stage 3 , elastic vibration of rock mass outside the fracture zone (region III). Blasting shock wave after the first two stages of rapid attenuation, vibration wave velocity attenuation to seismic waves, only can cause rock elastic vibration, basically no plastic deformation. At the same time, it is found that the influence range III $>>$ II $>$ I, and the development speed and influence range of blasting cracks are far smaller than the propagation range of stress waves, so the talus slope is only affected by seismic waves when it is far from the blasthole.

After the stress wave reaches the tunnel face, the compression wave reflects the reflected tensile stress wave and propagates to the source of the compression wave, forming a tensile stress zone within a certain range of the interface. Its strength is sufficient to break the rock, resulting in multi-layered lenticular delamination (Fig. 11). Throwing objects eventually accumulate near the tunnel face under the action of impact force, air resistance and self-weight. The vibration velocity of talus slope at different time after blasting is shown in Fig. 12. After blasting, the circular wave front rapidly expands outward and produces refraction and reflection at the interface (Fig. 12 (a) $\sim(c)$ ). The larger velocity in the lower part will transfer to the upper slope (Fig. 12(d) (f)), and gradually stabilize from bottom to top. 


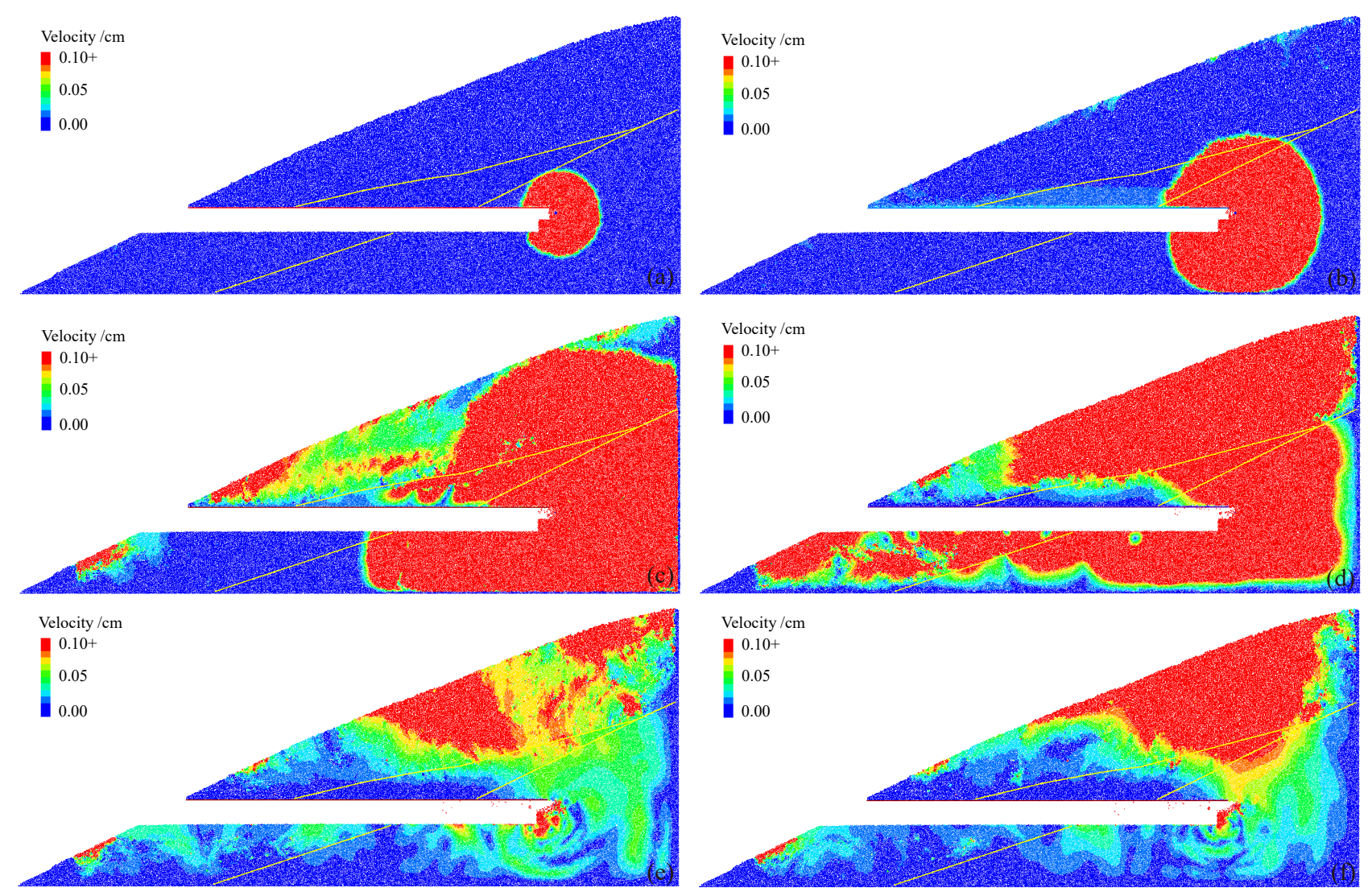

Fig. 12 Velocity of talus slope at different time (expressed in red when it's greater than $0.1 \mathrm{~cm} \times \mathrm{s}^{-1}$, and the following is the same; (a) (f) represent $t$ is $0.1 \times 10^{4}$ steps, $2 \times 10^{4}$ steps, $0.54 \times 10^{4}$ steps, $2.6 \times 10^{4}$ steps and $5.4 \times 10^{4}$ steps respectively)

The bedrock is located in crushing zone and fracture zone during blasting. The accumulation horizon is in the elastic vibration zone, and may be in the fracture zone when the explosive quantity is large or near the explosion center. The talus is in the elastic vibration zone (the tunnel entrance generally doesn't explode). When the stress wave or seismic wave passes through the interface, the interface reflection and refraction should also be considered. There are two main interfaces between the accumulation horizon and the bedrock, and between the talus and the accumulation horizon.

Fig. 12 shows that the wave front after blasting is similar to circular, and develops to the slope surface after two refractions at the interfaces, and finally the wave front is tangent to the surface of the slope. In general, when the wave front is circular, the tangent point is the place arrived firstly. From Fig. 12 (a) (c), the wave front and the cut point of the interface are connected, and the main direction of slope development shown in Fig. 13 is obtained. The cut point is just above the vertical explosion center, which is another reason for the first arrival of the point. By amplifying the velocity of rock at the initial point (Fig. 14), it's found that the vibration direction is consistent with the propagation direction in

Fig. 13, and isn't vertically upward, so it's not caused by the reason two.

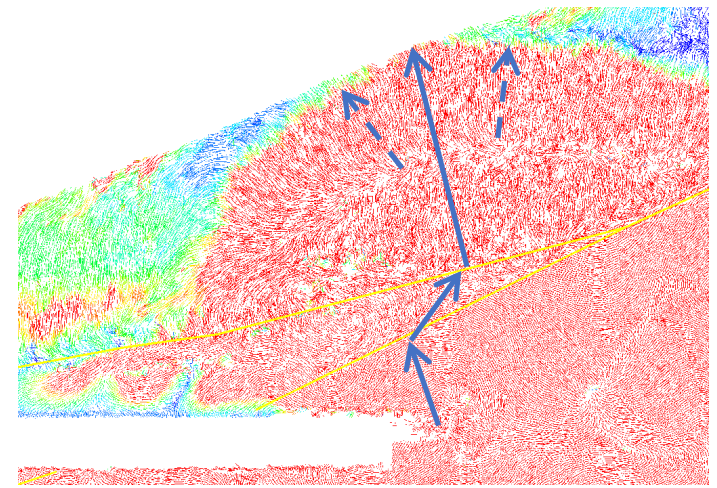

Fig. 13 Propagation direction of seismic wave

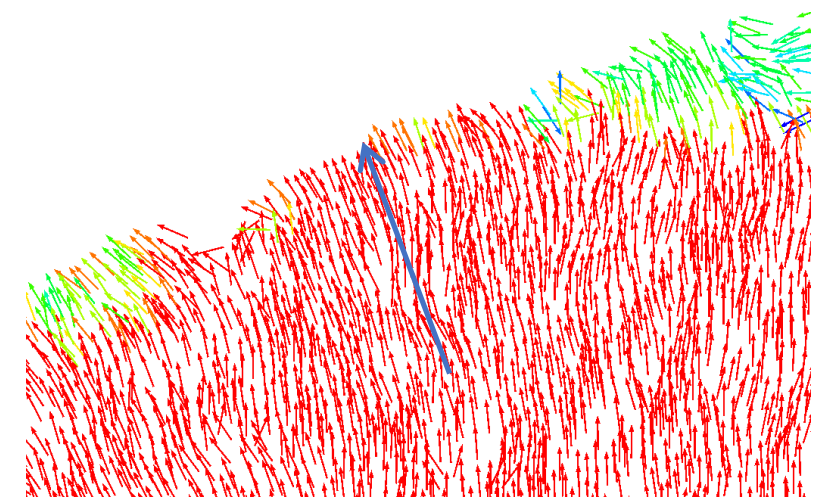

Fig. 14 Vibration direction of rock on the surface of the slope

After multiple reflections and refractions inside the slope, especially the initial compressive stress wave is reflected 
as tensile wave at the interface, and when the tensile wave reaches the surface of the explosion hole, it will be reflected as compressive wave. The rock particles between the explosive and the free surface are all accelerated, and the deep rock crack stops expanding due to the unloading of tensile waves. A very complex dynamic stress field is formed under the combined action of compression wave, tensile wave and explosion gas pressure in the explosion chamber (Fig. 12 (d) $\sim(\mathrm{f})$ ). The rock between the hole and the tunnel face uplifts, ruptures and bulges, and finally the tunnel face forms a throwing funnel and the throwing objects accumulate near the tunnel face.

After blasting, the bedrock quickly becomes calm, but the talus slope is difficult to be stable for a long time, and the local talus slope is in a metastable state. It is easy to form collapse holes under the weakening or slight disturbance of the contact, and even cause the collapse of the talus slope. It shows the discontinuity and anisotropy of rock mass, and also reflects the rationality and necessity of irregular particle modeling.

Through the above analysis, it can be seen that the vibration process of bedrock and talus slope reflected by the numerical simulation based on particle discrete element is consistent with the vibration effect and stress wave propagation law after blasting in practice, indicating that it is feasible to use this method to simulate the vibration process.

\subsection{Eddy current phenomenon of rock}

After the explosion, as shown in Fig. 15(a), two concentric circular wave fronts appear around the blasthole.
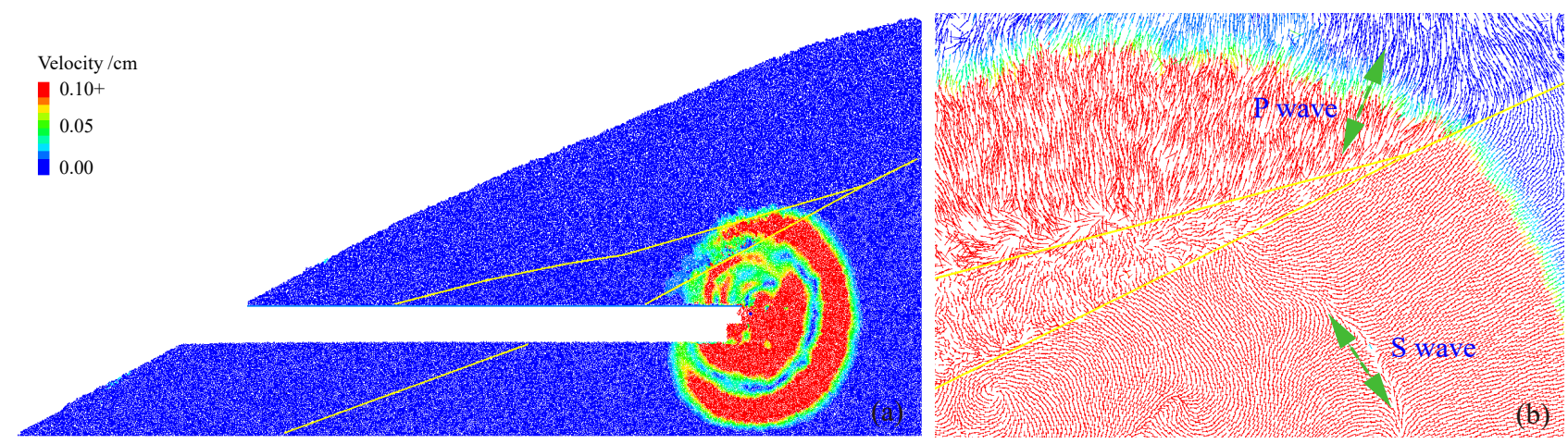

Fig. 15 Front of $P, S$ wave $\left(t=3.0 \times 10^{3}\right.$ steps; (a) and (b) represent entirety of the model and enlarged model respectively)

After velocity amplification, as shown in Fig. 15 (b), the first wave front move along the wave front propagation direction, and the second wave front rock particles move along the vertical propagation direction, corresponding to the $\mathrm{P}$ wave and $\mathrm{S}$ wave respectively. Before blasting, the particles are close to be static state and the whole movement direction is disordered. After blasting, the movement direction of rock changed from disorder to orderly state driven by stress wave or seismic wave. The vibration velocity was similar to the ripple produced by the calm lake surface falling into the stone. When encountering obstacles, refraction and reflection occurred.

The vibration velocity vector diagram of some stages of the vibration process in Fig. 16 shows that the rock diffuses outward driven by blasting, and the seismic wave diffuses to both sides after reaching the slope surface, which is similar to the phenomenon of fluid vortex. When the kinetic energy decays to less than enough to push the particles upward vibration, the particles change the direction of vibration. Similar multiple cycles induce wave attenuation of velocity. Finally, after multiple refraction and reflection at the interfaces, the direction of particle vibration is gradually disordered, and it is still again after the dissipation of elastic wave.

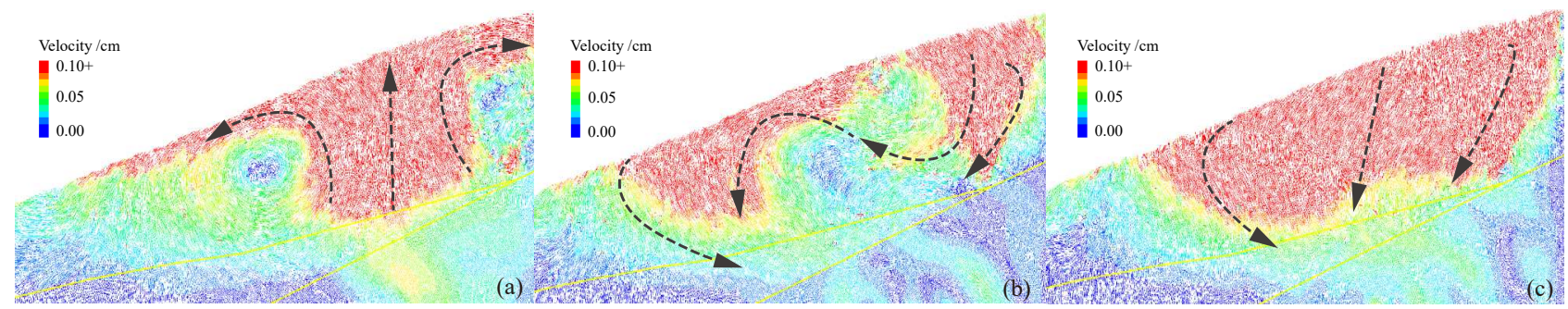

Fig. 16 Eddy current of the talus slope ((a), (b) and (c) represent $t$ is $5.8 \times 10^{4}, 6.1 \times 10^{4}$ and $6.3 \times 10^{4}$ steps respectively) 


\subsection{Potential sliding surfaces and local unstable structures}

Compared with the rock slope with great integrity, the displacement of talus slope with discontinuity is larger under the same condition, and the slight movement of the lower rock block may cause large-scale collapse. Figs. 17(a) $\sim(d)$ are the images of slope displacement in partial time.

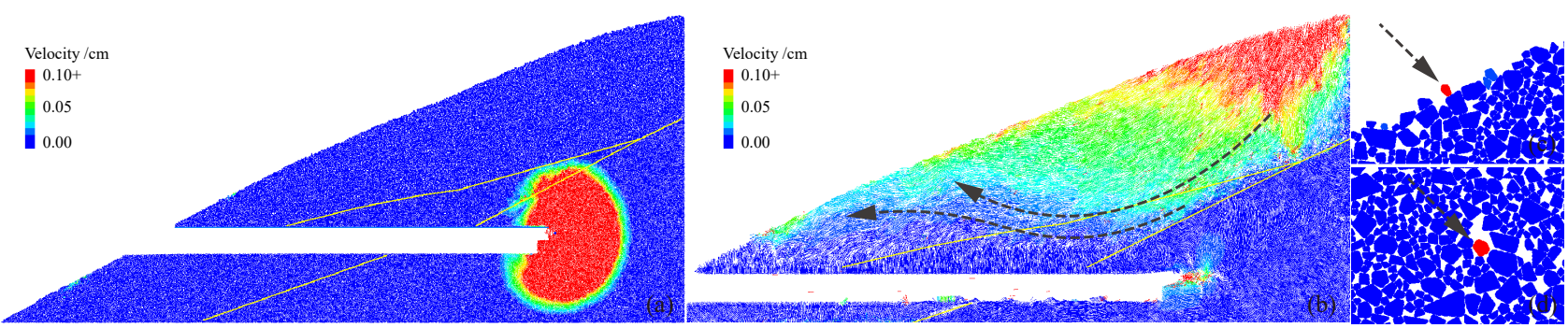

Fig. 17 Displacement of talus slope((a) represent $t$ is $2.0 \times 10^{3} \mathrm{steps}$; (b), (c)and (d) represent $t$ is $1.4 \times 10^{5} \mathrm{steps}$, and represent unstable rock blocks on the surface and inside of the slope respectively)

Similar to the velocity, the rock particles move outward radially in concentric circles after blasting, reflecting and refracting at the interfaces. From the displacement of the slope at the time step, $1.4 \times 10^{5}$ (Fig. 17(b)), it can be seen that the displacement from the foot to the top of the slope increases gradually, mostly less than $0.6 \mathrm{~cm}$, and there is a potential arc slip surface at the interface between the talus slope and the bedrock, the talus slope and the accumulation horizon. There is a large displacement of individual rock in the slope surface and inside (Fig. 17(c) (d)), reaching about $2.0 \mathrm{~m}$.

\section{Vibration attenuation law and vibration velocity evaluation of talus slope}

\subsection{Evaluation of peak vibration velocity}

The relatively mature blasting vibration velocity evaluation formula is Sadovsky formula, which is widely adopted in China. The attenuation law of vibration velocity in homogeneous hard rock mass with good continuity under blasting vibration is well fitted, but the applicability in loose rock slope needs further research. Domestic and foreign scholars use dimensional analysis and engineering verification methods to study the dynamic response law and stability of rock slope under blasting from slope effect (Guo et al. 2001), elevation (Tang et al. 2007) and topography (Tang and Li 2011). Previous studies have shown that the peak velocity $V$ is not only related to the distance between the blasting center and the slope surface, $R$, dosage, $Q$, and geological conditions, $K$, but also related to the elevation difference $H$, horizontal distance $D_{h}$ and other factors. Therefore, the Sadovsky family formula shown in Table 4. where $K$ represents the blasting conditions coefficient, $\alpha$ is the geological condition coefficient, $\gamma$ is the relative slope influence factor, and $\beta$ is the elevation influence factor.

Table 4 Attenuation formulas of vibration velocity

\begin{tabular}{cccc}
\hline Order & Time & Source & Formula \\
\hline 1 & 1945 & USSR (Sadovsky, 1945) & $V=K\left(Q^{1 / 3} / R\right)^{\alpha}$ \\
2 & 1955 & Europe (Wang et al. 2004) & $V=K\left(Q / R^{2}\right)^{\alpha}$ \\
3 & 1958 & Sweden (Wang et al. 2004) & $V=K Q^{1 / 2} / R^{3 / 4}$ \\
4 & 1966 & USA (Wang et al. 2004) & $V=K\left(Q^{1 / 2} / R\right)^{\alpha}$ \\
5 & 1976 & Japan (Wang et al. 2004) & $V=K Q^{3 / 4} / R^{2}$ \\
6 & 1988 & Zhu Chuantong (1988) & $V=K\left(Q^{1 / 3} / R\right)^{\alpha}\left(Q^{1 / 3} / H\right)^{\beta}$ \\
7 & 2004 & Hu and Wu (2004) & $V=K\left(Q^{1 / 3} / R\right)^{\alpha}\left(R / D_{h}\right)^{\beta}$ \\
8 & 2007 & Standard (2007) & $\left.\left.V=K\left(Q^{1 / 3} / D_{h}\right)^{\alpha}\right) Q^{1 / 3} / H\right)^{\beta}$ \\
9 & 2011 & Tang et al. (2011) & $V=K_{1} K_{2}\left(Q^{1 / 3} / R\right)^{\alpha}(H / R)^{\beta}$ \\
10 & 2020 & He et al. (2020) & $V=K\left(Q^{1 / 3} / D_{h}\right)^{\alpha}\left(R / D_{h}\right)^{\beta}\left(H / D_{h}\right)^{\gamma}$ \\
\hline
\end{tabular}

In addition, including the Swedish scholar, Langfors, in 1958, the European scholar, P. B. Attewell et al., in 1955, the American Bureau of Mines, J. R. Devine et al., in 1966(Wang et al. 2004), the Japanese Asahito Industrial Co. Ltd., 
in 1976 (Table 4), and the UK, India et al. (Lei 2015), the attenuation formula of peak vibration velocity adapted to the country was proposed.

To eliminate the local limitations of formulas, a more general Sadovsky family formula can be derived from table 4:

$$
V=K Q^{\alpha} R^{\beta} D_{h}^{\gamma} H^{\xi}
$$

Where $V, K, Q, R, D_{h}$ and $H$ have the same meaning as before. $\alpha, \beta, \gamma$ and $\xi$ are coefficients related to blasting conditions, which are obtained through field blasting or experience.

The velocity is not only related to the elastic wave caused by blasting, but also affected by gravity. Unlike continuous rock, there is no cohesive force between rock blocks to prevent separation. If the vertical line between the blasting center and the slope surface is taken as dividing line of the dominant force. The peak velocity $V$ is dominated by the blasting load in the area below the boundary, by blasting load and gravity above the boundary and gradually transits to only gravity.

Below the dividing line, the elastic wave caused by blasting and gravity result in the downward compaction of the rock. Because the rebound elastic force is weaker than the initial force. The overall performance is compression state. The properties of rock mass are similar to those of continuous rock mass. Above the dividing line, the elastic wave caused by blasting will also cause block compaction, but the direction is upward. When rebounding, gravity dominates the mechanical behavior of rock. Therefore, the overall performance is tensile state. Blocks fall under the action of gravity and elastic potential energy accumulated by elastic wave when compress rock blocks. In addition, the downward displacement of the upper rock block is intensified due to the compaction of the lower part. Therefore, the rock mass above the dividing line reflects its discontinuous characteristics and enlarges the blasting effect.

According to 17 groups data on site, the velocity of each direction are fitted by domestic formula in table 4(Fig. 18).

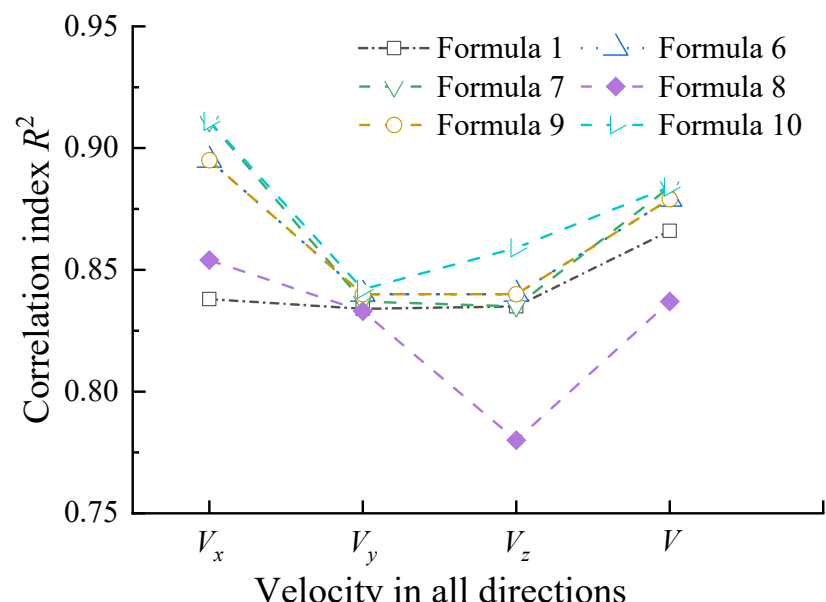

Fig. 18 Correlation index $R^{2}$ diagram fitted by different formulas

The goodness of fit is judged by the correlation index $R^{2}$. From Fig. 18, except for the poor fitting of Formula 8 , the $R^{2}$ values of the other formulas in different directions are greater than 0.83 , and the overall fitting effect is good. Due to the talus slope structure is loose, discrete, and affected by the difference of sample data, resulting in the overall fitting degree is not high. Since the monitoring positions are all below the line, the positions above need to be further verified by numerical simulation.

\subsection{Vibration attenuation law of talus slope}

The talus slope is mainly composed of two-phase or three-phase medium. The seismic wave propagates in slope skeleton itself and the interstitial fillings such as gas and liquid, which leads to the attenuation of vibration wave, which is the main influencing factor of attenuation. Blasting vibration causes talus slope particles to collide with each other. At the same time, the rapid movement of gas and liquid molecules produces large amounts of heat and absorbs vibration waves. Therefore, vibration frequency gradually weakened, and the waveform gradually dispersed. The faster the vibration frequency is, the faster the vibration attenuation is.

In order to further explore the vibration and seismic wave attenuation law of the talus slope, the monitoring of the 
horizontal right and vertical upward of the blasthole, the horizontal position of the upper part of the tunnel and the slope surface are selected to analyze the vibration velocity and displacement response.

Fig. 19 and Fig. 20 are the time-history curves of velocity and displacement of horizontal right monitoring points. The velocity and displacement fast first and then slow, like wave attenuation.

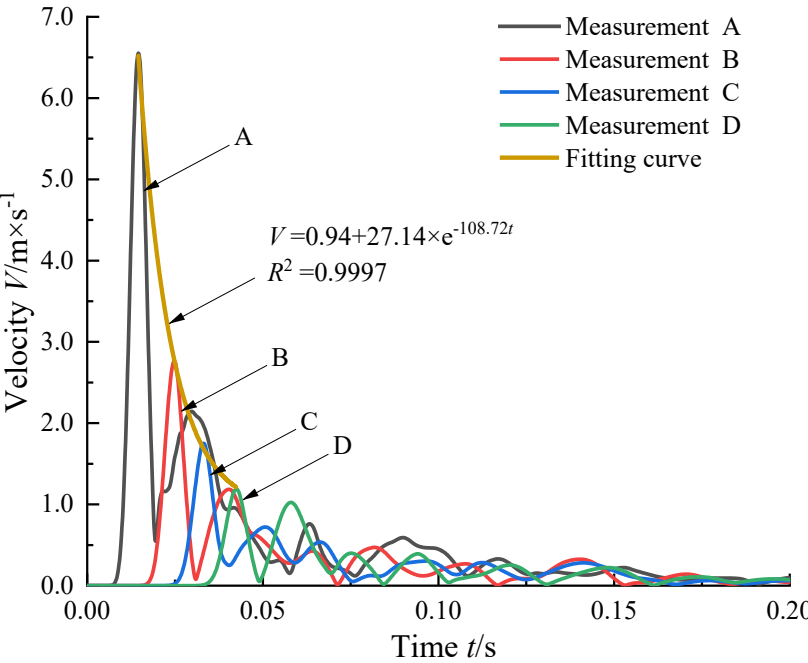

Fig. 19 Velocity time-history curve in $X$ positive direction

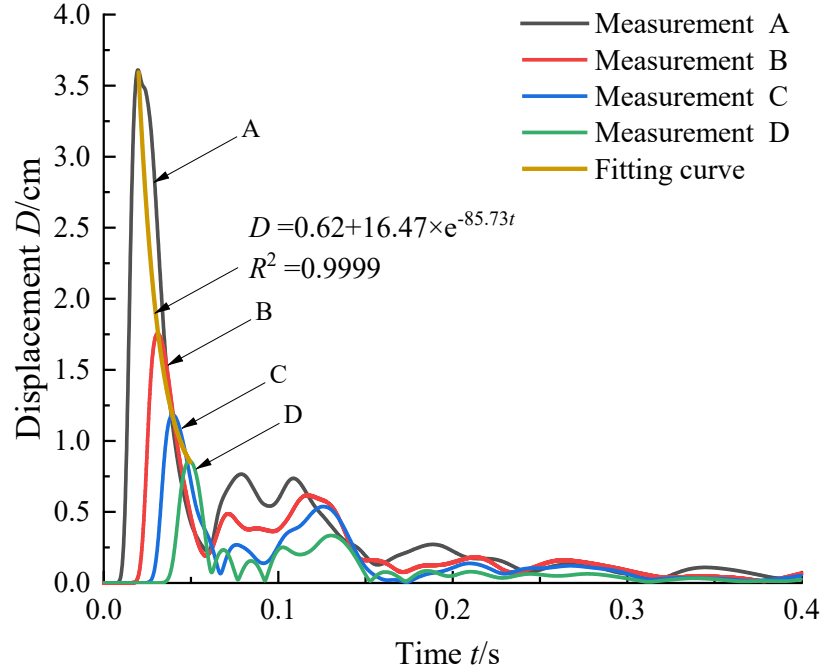

Fig. 20 Displacement time-history curve in $X$ positive direction

The difference is that the rock velocity tends to be static after a certain time, and the displacement of the closer monitoring point tends to be nonzero due to the residual deformation.

The descending exponential function (Formula 16) is used for fitting. The fitting results is good. The velocity and displacement response of horizontal monitoring points above the tunnel are similar.

$$
y=a_{0}+b_{0} \times e^{-c_{0} x}
$$

In the formula $y$ is velocity or displacement. $x$ is time or distance. $a_{0}, b_{0}$ and $c_{0}$ are constants. The greater the $c_{0}$, the faster the attenuation.

The peak velocity and peak displacement responses in the vertical direction are shown in Fig. 21 and Fig. 22. The peak velocity in the vertical direction also decays exponentially with the increase of elevation (time), and the attenuation gradually slows down. The peak displacement of accumulation horizon layer increases exponentially with the increase of elevation (time), and transits to linear growth in talus.

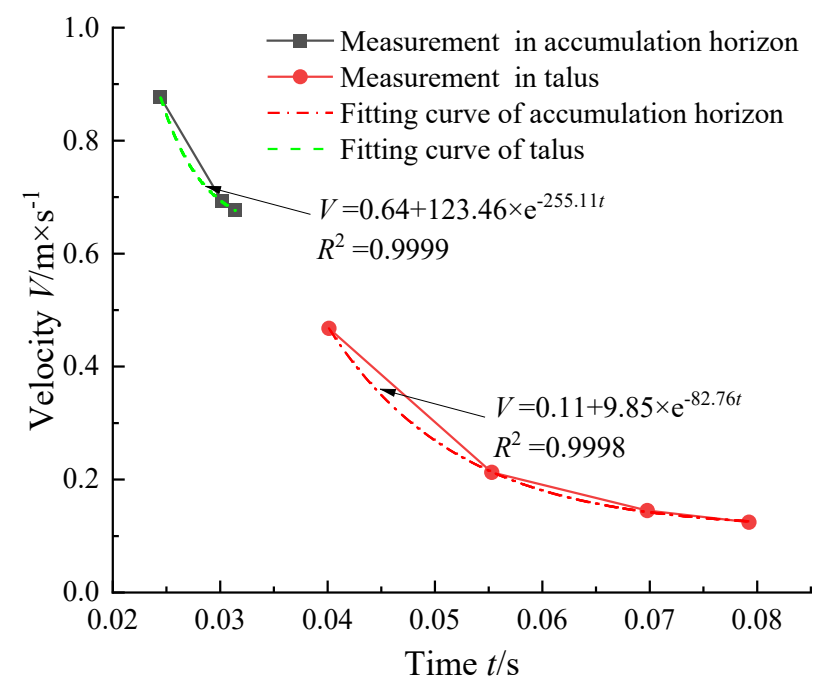

Fig. 21 Attenuation curve of velocity in vertical direction

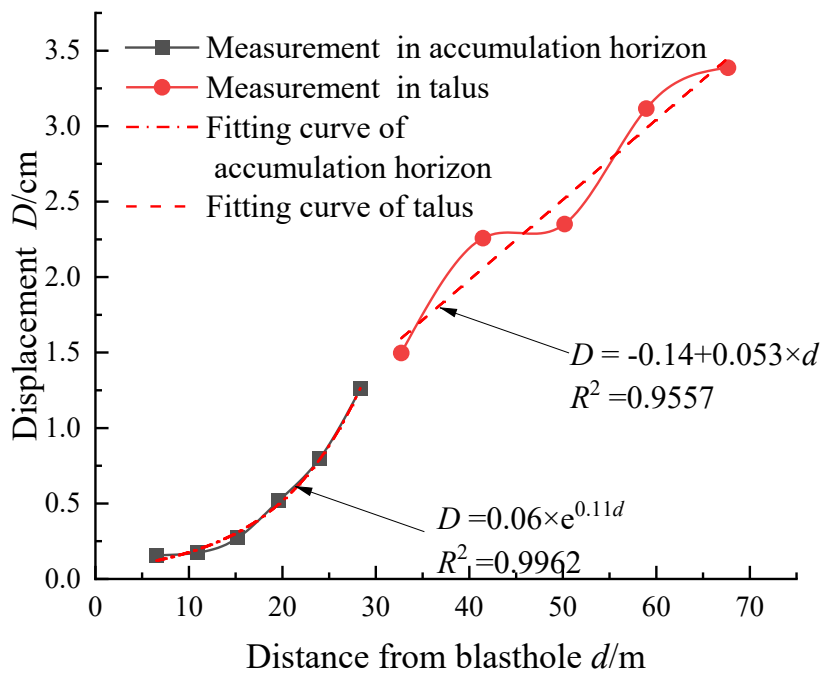

Fig. 22 Attenuation curve of displacement in vertical direction 
displacement of the surface rock increases linearly with the distance from the tunnel entrance. The peak velocity increases exponentially with the increase of distance. The velocity of the tunnel entrance has an amplification effect, which is consistent with the actual phenomenon.

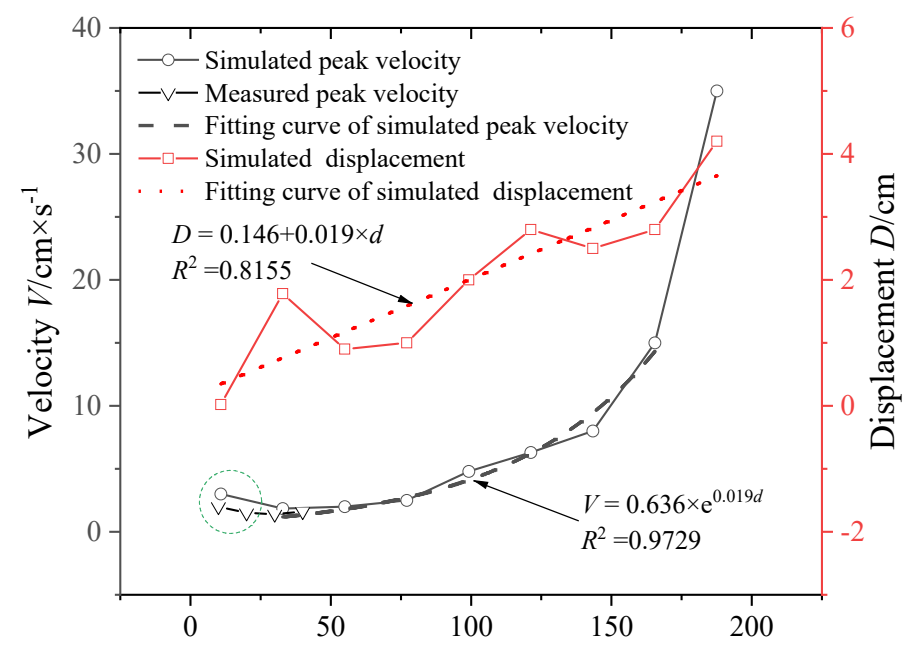

Fig. 23 Velocity and displacement attenuation curve of slope surface

According to the fitting results, when the blasting position and blasting charge are fixed, the peak vibration velocity of talus slope meets the exponential growth law:

$$
V=K e^{\gamma d}
$$

In the formula, $V, K$ means the same as above, $d$ is the horizontal distance between a point on the slope and the tunnel entrance. $\gamma$ is the coefficient.

Unlike table 4 above, the measured and simulated velocity values below the dividing line (correlation index $R^{2}>0.9$ ) basically meet the Sadovsky formula. The difference above the dividing line is large, which is exponential growth, and the overall fitting is good. The measured peak vibration velocity is between the exponential curve and the simulated value. Similarly, the tunnel entrance amplification effect is not well represented at present.

In addition to the amplification effect of tunnel entrance, the formula (17) and Sadovsky family formulas can't be better expressed, the measured and simulated values of velocity near the entrance (correlation index $R^{2}>0.9$ ) basically meet formulas, but the difference increases gradually along the slope. The measured peak vibration velocity is between the exponential curve and the simulated value.

\section{Conclusions}

(1) The size of the rock in the talus meets the characteristics of Gaussian distribution after Johnson transformation. The overall size of rock blocks in the field does not change significantly with the slope height, which is relatively small at the toe and top of slope.

(2) The process of rock breaking by blasting, vibration of the talus slope, and the propagation law of stress wave reflected by the numerical simulation based on two-dimensional particle discrete element are consistent with the actual situation, which shows that this method is feasible to simulate the vibration process of talus slope subjected to blasting. The irregular particles close to the actual shape can better reflect the real process and overcome the shortcomings of continuum theory.

(3) The peak velocity and peak displacement in the horizontal direction decay exponentially. The peak velocity in vertical direction decreases exponentially with the increase of elevation or time, and the attenuation speed slows down gradually. The peak displacement in vertical direction, of accumulation horizon, increases exponentially with the increase of elevation (or time), and transits to linear growth in talus.

(4) With the increase of the distance from the tunnel entrance, the peak displacement of the rock on the slope surface increases linearly and the peak velocity increases exponentially. There is an amplification effect on the velocity and 
acceleration at the entrance, which is consistent with the phenomenon actually monitored.

(5) The discontinuity of the talus slope is the direct reason that the peak velocity of slope increases exponentially and gradually doesn't meet the Sadovsky family formula upward.

Acknowledgements The authors gratefully acknowledge the respected editors and the anonymous referees for their suggestions in this article. Special thanks are given to financial supports provided by the Foundation of Key Laboratory of Geological Hazards on Three Gorges Reservoir Area, Ministry of Education (Grant No. 2020KDZ06) and the Major Special Fund for China University of Mining and Technology(2018WHCCO5).

\section{References:}

Bahrani N, Valley B, Kaiser PK, Pierce, M (2011) Evaluation of PFC2D Grain-Based Model for simulation of confinement-dependent rock strength degradation and failure processes. Proc.,45th US Rock Mechanics / Geomechanics Symposium.

Cho N, Martin CD, Sego DC (2007) A clumped particle model for rock[J]. International Journal of Rock Mechanics \& Mining Sciences 44(7):997-1010. https://doi.org/10.1016/j.ijrmms.2007.02.002

Cundall P A, Strack ODL (1979) A discrete numerical model for granular assemblies. Géotechnique 29(1): 331-336. https://doi.org/10.1680/geot.1980.30.3.331 Edmans BD, Sinka IC (2020) Numerical derivation of a normal contact law for compressible plastic particles. Mechanics of Materials 146: 103297.1-103297.19. 10.1016/j.mechmat.2019.103297

Guo XB, Xiao ZX, Zhang ZC (2001) Slope effect of blasting vibration. Chinese Journal of Rock Mechanics and Engineering 1:83-86. https://doi.org/10.3321/j.issn:1000-6915.2001.01.019 (in Chinese)

Hazzard JF, Young RP (2004) Numerical investigation of induced cracking and seismic velocity changes in brittle rock. John Wiley \& Sons, Ltd, 31(1): 16041607. https://doi.org/10.1029/2003GL019190

He J (2016) Stability analysis of talus slope. Beijing, China University of Geosciences (Beijing). https://oi.org/CNKI:CDMD:2.1016.184274 (in Chinese)

He L, Zhong DW, Li P, Song K, Si JF (2020) Vibration prediction and energy analysis of slope under blasting load in underpass tunnel. Explosion and Shock Waves 40 (7): 108-117. https://doi.org/10.11883/bzycj-2019-0255 (in Chinese)

Hertz HR (1881) Ueber die Vertheilung der Electricität auf der Oberfläche bewegter Leiter. John Wiley \& Sons, Ltd 249(6):266-275.

Hu G, Wu YL (2004) A new method of the control of the earthquake vibration caused by explosive. Coal Technology (4): 104-106. Hu HT, Liu YJ (2003) Study on fluid dynamics theory of high speed remote landslide. Southwest Jiaotong University. (in Chinese)

Lei Z (2015) Research and application on nonlinear prediction methods of bench blasting vibration effect. Beijing, China University of Mining and Technology (Beijing). https://doi.org/ CNKI:CDMD:1.1015.304641 (in Chinese)

Ministry of water resources of the people's Republic of China (2007) DL/T 5389-2007 Technical code for construction of rock foundation excavation of hydraulic structures. Beijing, People's Communications Press. (in Chinese)

Nabipour A (2013) Experimental and numerical study of ultrasonic monitoring of hydraulic fracture propagation. Perth, Curtin University.

Peng C, Liu WR, Wang Z (2020) Parameter Checking Method of Large Scale Particle Model. Geotechnical and Geological Engineering 39(2):1533-1540. https://doi.org/ 10.1007/s10706-020-01574-1

Qi CZ, Qian QH (2009) Basic problems of dynamic deformation and fracture of rock mass. Beijing: Science Press. (in Chinese) Sadovsky (1945) Experimental study on mechanical effect of blast wave. Leningrad: Sciences Press of Soviet Academy, 1-43. (in Russia)

Scaringi G, Fan XM, Xu Q, Liu C, Ouyang CJ, Domenech G, Yang F, Dai LX (2018) Some considerations on the use of numerical methods to simulate past landslides and possible new failures: the case of the recent Xinmo landslide (Sichuan, China). Landslides 15(7): 1359-1375. https://doi.org/10.1007/s10346-0180953-9

Shi JJ, Zhang W, Wang W, Sun YH, Xu CY, Zhu HH, Sun ZX (2021) Randomly generating three-dimensional realistic schistous sand particles using deep learning: Variational autoencoder implementation. Engineering Geology 291(1-2):106235. https://doi.org/10.1016/j.enggeo.2021.106235

Shugar DH, Jacquemart M, Shean D, Bhushan S, Upadhyay K, Sattar A et al. (2021) A massive rock and ice avalanche caused the 2021 disaster at Chamoli, Indian Himalaya. Science (New York, N.Y.) 373(6552):300-306. https://doi.org/10.1126/SCIENCE.ABH4455

Su D, Wang X (2021) Fourier series-based discrete element method for two-dimensional concave irregular particles. Computers and Geotechnics 132. https://doi.org/10.1016/j.compgeo.2020.103991 
Tang H, LI HB, Jiang PC, Wang XW, Li JR (2007) Experimental study on effect of topography on propagation of blasting waves [J]. Chinese Journal of Rock Mechanics and Engineering 9: 1817-1823. https://doi.org/ 10.3321/j.issn:1000-6915.2007.09.012 (in Chinese)

Tang H, Li HB (2011) Study of blasting vibration formula of reflecting amplification effect on elevation. Rock and Soil Mechanics 32 (3): 820-824. https://doi.org/ 10.16285/j.rsm.2011.03.026 (in Chinese)

Wang XD, Ruan YF, Li Q, Wang F (2014) Situation \&amp; prospect of research on Talus Slope. Proc., 2nd International Conference on Advances in Computational

Wang XG, Yu YL, Liu DZ (2004) Enforceable handbook of safety regulations for blasting[M]262:131-133. (in Chinese)

Wang BX, Cheng PF, Zheng YX, Zhou FF (2020) Attenuation law of stress wave in granular particles. Chinese Journal of High Pressure Physics 34(4):100-107. https://doi.org/10.11858/gywlxb.20200508 (in Chinese)

Wang ZJ, Ruiken A, Jacobs F, Ziegler M (2014) A new suggestion for determining 2D porosities in DEM studies. Geomechanics and Engineering 7(6): 665-678. https://doi.org/ 10.12989/gae.2014.7.6.665 Xu HB (2017) Study on Dynamic Response of Talus Tunnel Entrance and Talus Slope under Blasting Vibration. Chengdu, Southwest Petroleum University. https://doi.org/10.27420/d.cnki.gxsyc.2017.000099 (in Chinese) Xu M, Song EX, Shen ZP, Zhu BQ, Yan JK, Nie YP, Wu B, Wang H, Fu JY (2019) Key techniques in geotechnical treatment of a large karst depression for fast. China Civil Engineering Journal 52 (3): 87-99. https://doi.org/10.15951/j.tmgcxb.2019.03.009 (in Chinese) Yang JX, Shi C, Wang SN, Zhang CH (2019) Numerical simulation verification of blasting failure effect in rock mass with particle flow code. Journal of Disaster Prevention and Mitigation Engineering 39 (2):217-226. https://doi.org/10.13409/j.cnki.jdpme.2019.02.004 (in Chinese) Zener C (1941) The Intrinsic Inelasticity of Large Plates. Modern Language Review 59(8):669-673. https://doi.org/ 10.1103/PhysRev.59.669 Zhang F, Zhou LX, Sun X, Yuan P. (2020) A microscale shear wave velocity model of earth-rock aggregate. Annales de Chimie - Science des Matériaux 44(3):223229. https://doi.org/10.18280/acsm.440310 Zhang YL, Liu ZB, Shi C, Shao JF. (2018) Three-dimensional Reconstruction of Block Shape Irregularity and its Effects on Block Impacts Using an EnergyBased Approach. Rock Mechanics and Rock Engineering 51(4):1173-1191. https://doi.org/ 10.1007/s00603-017-1385-x Zheng H, Niu WQ, Mao WW, Li LH, Wang FW, Huang Y. (2021) Mechanics of granular material and the application in engineering geology. Journal of Engineering Geology 29(1):12-24. https://doi.org/10.13544/j.cnki.jeg.2021-0017 (in Chinese) Zhu CT, Liu HG, Mei JY (1988) Selection of formulas for propagation of seismic wave parameters along slopes. Blasting (2): 30-31. (in Chinese) Zhu DP, Xie CJ, Xu HB (2020) Experimental study on dynamic response of surrounding rock of entrance section of tunnel under blasting vibration. Chinese 


\section{Supplementary Files}

This is a list of supplementary files associated with this preprint. Click to download.

- annex.rar 